\title{
CUFF INFLATION TO AID NASOTRACHEAL INTUBATION USING THE C-MAC VIDEO LARYNGOSCOPE- A COMPARISON OF TWO TYPES OF ENDOTRACHEAL TUBES
}

\author{
Ashok M. S1, Deepali N. V²
}

1MBBS, Department of Anaesthesia, M. S. Ramaiah Medical College and Hospital, Bangalore, Karnataka, India. 2MBBS, Department of Anaesthesia, East Point Medical College and Hospital, Bangalore, Karnataka.

\section{ABSTRACT}

\section{BACKGROUND}

Nasotracheal intubation offers the head and neck surgeons more scope for surgical manoeuvres.

Aim: We decided to study the success rate of nasotracheal intubation with the aid of an inflated cuff of an ET tube in the oropharynx and to avoid the usage of forceps using a video laryngoscope and also to compare the above technique using two different tubes i.e. PVC ET tubes and flexometallic ET tubes.

\section{MATERIALS AND METHODS}

Sixty consenting adults were randomly allotted to be intubated nasally with a conventional PVC ET tube or flexometallic tube using a C-MAC video laryngoscope. The time taken, any other manoeuvres used, failure rate and any complications were noted. The sample size was calculated to be 30 patients in each group. Sample size was calculated in order to achieve a power of $80 \%$ and considering alpha error of $10 \%$.

\section{RESULTS}

A statistically significant increase in the success rate of nasotracheal intubation with the aid of an inflated cuff of an ET tube and with a C-MAC video laryngoscope were noted in both groups, which was the primary outcome studied. In PVC group and in flexometallic group, 8 patients and 13 patients were intubated with the aid of cuff inflation using a C-MAC video laryngoscope respectively, 22 patients and 17 patients were intubated without the aid of cuff inflation respectively.

\section{CONCLUSION}

The usage of C-MAC video laryngoscope for nasotracheal intubation of PVC and Flexometallic ETT reduced the grade of difficulty and provided easy navigability of ETT from laryngeal inlet to trachea and also decreased the amount of time taken for intubation significantly.

\section{KEY WORDS}

C-MAC, Cuff Inflation, PVC ETT, Flexometallic ET.

HOW TO CITE THIS ARTICLE: Ashok MS, Deepali NV. Cuff inflation to aid nasotracheal intubation using the C-MAC video laryngoscope- a comparison of two types of endotracheal tubes. J. Evolution Med. Dent. Sci. 2018;7(39):4268-4271, DOI: $10.14260 /$ jemds/2018/952

\section{BACKGROUND}

Softer flexometallic Endotracheal Tubes (ETT) compared to regular PVC ET tubes are more difficult to navigate in oropharynx during Nasotracheal Intubation (NTI). Cuff inflation has been used to aid nasotracheal intubation both in blind NTI and in direct laryngoscopy for NTI, but the success rate for NTI using C-MAC guided video laryngoscopy has been not studied. It is a randomised controlled trial. We compared the ease of navigability and time taken and nasal injury between regular PVC ET tubes and flexometallic ET tubes for successful NTI with the aid of cuff inflation with C-MAC guided video laryngoscope. Video laryngoscopes (VL) which work on the principles of indirect laryngoscopy have become popular in clinical practice. ${ }^{[1,2]}$ They provide a significantly better view of the larynx, which may be useful in difficult tracheal intubation scenarios. $[3,4,5,6]$

'Financial or Other Competing Interest': None.

Submission 18-08-2018, Peer Review 11-09-2018,

Acceptance 17-09-2018, Published 24-09-2018.

Corresponding Author:

Deepali N. $V$,

No. 5, Vishwadeep, $1^{\text {st }}$ Cross,

Chola Nagar, RT Nagar Post,

Hebbal, Bangalore-560032, Karnataka, India.

E-mail: deepali.navale@yahoo.com

DOI: $10.14260 /$ jemds $/ 2018 / 952$

\section{(c) $(1) \$$}

The key novel feature of these 'indirect' laryngoscopes is that they facilitate visualisation of the vocal cords without the need to align the oropharyngeal and tracheal axes.[7]

Kaplan and Berci introduced C-MAC ${ }^{\circledR}$ VL (Karl Storz, Tuttlingen, Germany) in 2003. It has been found to improve Cormack-Lehane (C-L) grading by 2 to 1 grade and possibly aid easier intubation. It provides both a direct laryngoscopic view and a small digital camera view that is displayed on the video screen in contrast to many previous VLs. [8]

Nasotracheal Intubation (NTI) is often required for head and neck surgeries like tonsillectomies. Direct laryngoscopy with the Macintosh blade with the use of Magill's forceps to direct Endotracheal Tube (ETT) into glottic opening, is timeconsuming and may lead to trauma to surrounding structures and damage ETT cuff.

In this study using video laryngoscope, we tried to manoeuvre the two types of endotracheal tubes, (PVC and Flexometallic) with cuff inflation (once it has passed through the nasopharynx) to pass through the glottic opening.

\section{Aims and Objectives}

The aim of this study was to study the success rate of two types of endotracheal tubes (PVC and Flexometallic) with CMAC video laryngoscope for nasotracheal intubation with the aid of an inflated cuff (once it has passed through the nasopharynx) and to avoid the usage of forceps. 


\section{MATERIALS AND METHODS}

It is a randomised controlled trial after IRB approval and written informed consent, 60 ASA grade I/II adults undergoing elective surgery and requiring ET intubation as part of anaesthetic management were enrolled in the study. The patients were allocated into two groups by computer generated numbers. Patients intubated with the aid of cuff inflation was taken as Group 1 and patients intubated without the aid of cuff inflation was taken as Group 2. Patients with bleeding diathesis, history of recurrent nasal obstruction or any nasal/ pharyngeal surgery, and those with anticipated difficult tracheal intubation were excluded. The sample size was taken for convenience during the study.

\section{Procedure}

The nasal cavity was prepared with topical preparation of $0.05 \%$ of xylometazoline drops twice into each nasal cavity. 30 minutes and 5 minutes before induction of anaesthesia along with 2 puffs of $10 \%$ lidocaine spray. Standard American Society of Anesthesiogists monitoring was instituted.

The patient in Operation Theatre received IV glycopyrrolate in a dose of $4 \mathrm{mcg} / \mathrm{kg}$, Inj. Midazolam 0.02 $\mathrm{mg} / \mathrm{kg}$, Inj. Fentanyl in a dose of $2 \mathrm{mcg} / \mathrm{kg}$ as premedication. Anaesthesia was induced with propofol $2 \mathrm{mg} / \mathrm{kg}$ followed by Inj. Atracurium $0.5 \mathrm{mg} / \mathrm{kg}$. After the induction dose of the above drugs, the patient was pre-oxygenated with $100 \%$ oxygen for three minutes with sevoflurane.9,10

The passage of endotracheal tubes, both PVC and Flexometallic were studied in three phases with regard to passage of ETT from nasopharynx to oropharynx, from oropharynx to laryngeal inlet and from laryngeal inlet to trachea. The grade of difficulty and time taken was noted in each of these three phases and also the volume of air required to aid the passage of ETT into trachea was noted.

In first phase of the procedure the nostril to be used for nasal intubation was lubricated with $2 \%$ lignocaine jelly, appropriate size of ETT was chosen as per the sex of the patient (Male- 7.5 and Female 7). The ease of passage of tube from nasal cavity to oropharynx was graded from 1 to 3.1easy to pass the ETT, 2- slight resistance encountered, 3- the ETT was not able to pass through selected nasal cavity and was inserted through other nasal cavity.

In second phase of the procedure, the ETT was passed from oropharynx into laryngeal inlet and the time taken for these and the grade of difficulty (grade 1 to 3 ) was noted. Grade 1- The ETT passes smoothly from oropharynx to laryngeal inlet with the aid of video laryngoscope. Grade 2Cuff inflation technique was used to align the ETT tube tip into the laryngeal inlet (The cuff of ETT once in oropharynx was gradually inflated with incremental volume of $4 \mathrm{~mL}$, maximum of $20 \mathrm{~mL}$ till the tip of ETT aligns with the laryngeal inlet. Once the ETT tip is in alignment of laryngeal inlet, the tip of ETT tube is pushed into the laryngeal inlet and then the cuff is deflated and the deflated ETT is further pushed into the trachea till the black line of ETT is at the level of vocal cords). Grade 3- Cuff inflation technique was not able to align the Tip of ETT to laryngeal inlet and Magill's forceps was used to guide the ETT into laryngeal inlet.11,12

In phase three, the ETT was passed from oropharynx into the laryngeal inlet and the time taken and the grade of difficulty was noted. Grade 1, ETT was passed smoothly with the use of video laryngoscopy. Grade 2 required slight manipulation of ETT (rotation and slight pressure was used).

After the completion of the intubation, it was confirmed by bilateral equal air entry and the tube was fixed and connected to anaesthesia ventilator and was maintained with oxygen, air and sevoflurane and intermittent atracurium.

Post-surgery, patient was extubated after being reversed with Inj. Glycopyrrolate $0.08 \mathrm{mcg} / \mathrm{kg}$ and Inj. Neostigmine $0.05 \mathrm{mg} / \mathrm{kg}$.

Post extubation patients were assessed for any complication like trauma to nasal cavity and persistent nasal bleed and patients were asked for any discomfort or pain in post-op area.

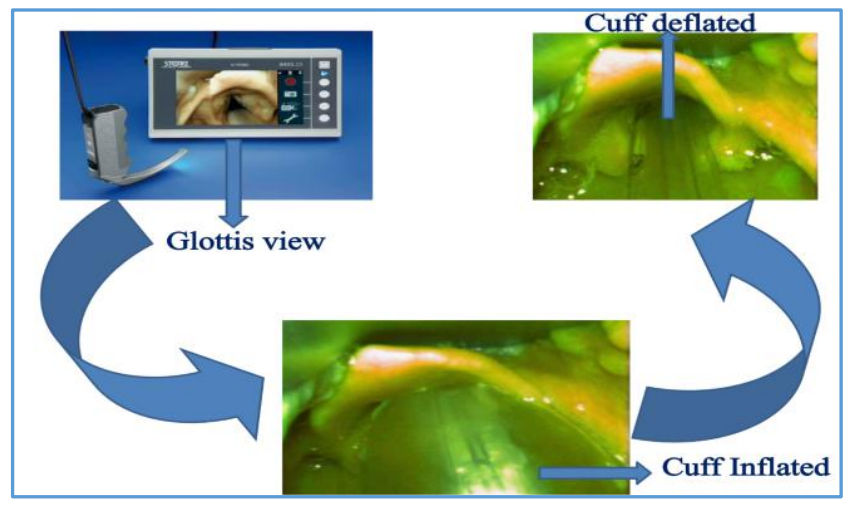

This study was done on 60 patients requiring nasal intubation. The failure rate, any complications and any other manoeuvres used were noted. Patients were randomly allocated to be intubated with a conventional PVC ET tube or flexometallic tubes (reinforced endotracheal tube). The sample size required was taken for convenience.

\section{Statistical Analysis}

All the quantitative parameters such as age, time taken etc. has been presented using descriptive statistics such as mean and standard deviation. All the qualitative parameters like grade of difficulty and if any complications etc. has been presented using frequency and percentage. Independent sample ' $\mathrm{T}$ ' test. The qualitative data was expressed by Chisquare test. Fisher's test was used. For statistical analysis, SPSS software version 25.0 was used.

\section{RESULTS}

The patient characteristics and demographic data were comparable in two groups.

In Group 1, i.e. PVC group, the grading of difficulty of 1,2 and 3 from nose to oropharynx (N0) were found in 50\%, 40\% and $10 \%$ patients respectively. Similarly, from oropharynx to laryngeal (OL) inlet was $40 \%, 53 \%$ and $3 \%$ respectively. The grading of difficulty of 1 and 2 for intubation from Laryngeal inlet to Trachea (LT) was $73.3 \%$ and $26.7 \%$ respectively.

In Group 2, i.e. Flexometallic group, the grading of difficulty of 1, 2 and 3 from N0 were found in 40\%, 43\% and $16.7 \%$ patients respectively. Similarly from OL inlet was $30 \%$, $63 \%$ and $6.7 \%$ respectively. The grading of difficulty of 1 and 2 for intubation from LT was $56.7 \%$ and $43.3 \%$ respectively.

The ' $\mathrm{p}$ ' value for intubating sequence from $\mathrm{NO}$ and $\mathrm{OL}$ was 0.646 and 0.56 respectively, which was statistically not significant and from LT it was 0.17 which was statistically significant. 
The duration of intubation with PVC ET was 33.33 secs and with flexometallic ET was 41.03 secs. The 'p' value was of 0.62 , which was statistically not significant.

Group 1 (PVC) had CL grade 1 and 2 in $46.7 \%$ and $63.3 \%$ patients respectively and in group 2 (WR), CL grade 1 and 2 was found in $53.3 \%$ and $36.7 \%$ patients respectively.

We did not encounter any major complications including epistaxis in our study population.

The usage of C-MAC video laryngoscope decreased the amount of time taken for intubation significantly in group 1 and 2 by 24.97 secs and 23.07 secs respectively.
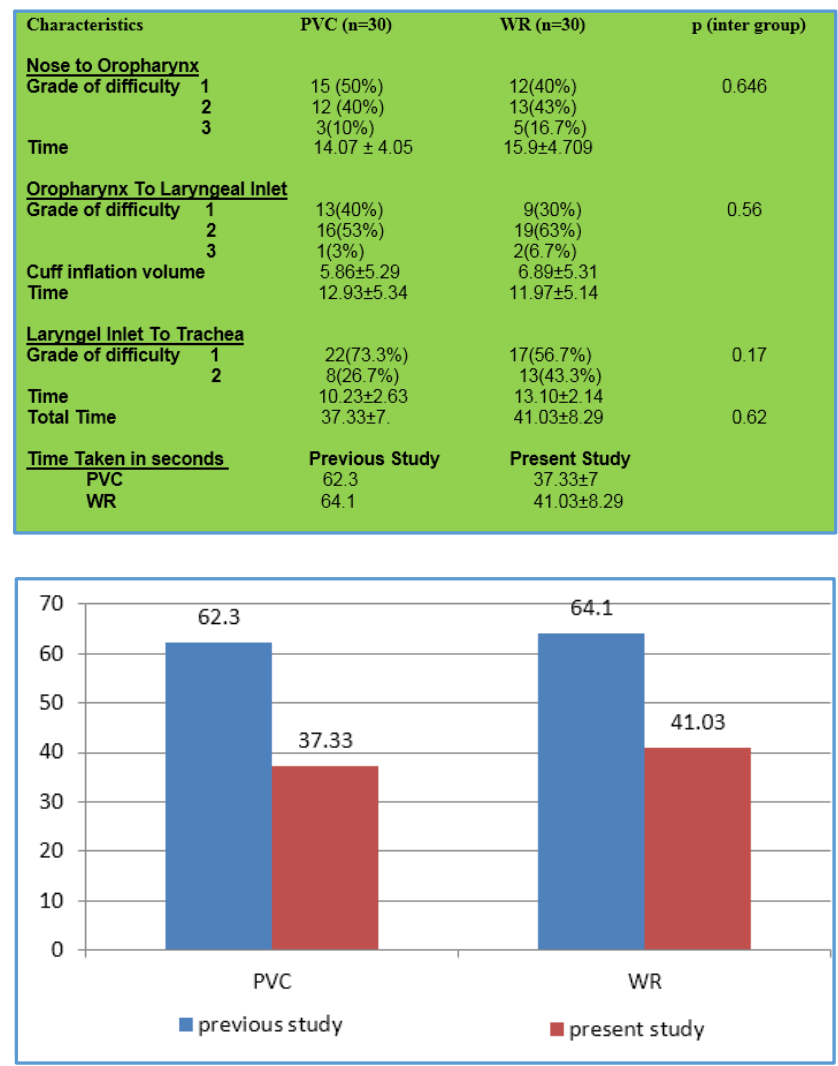

\section{DISCUSSION}

The study revealed that the cuff inflation technique improved the navigation of both the endotracheal tubes irrespective of their stiffness from the oropharynx into laryngeal inlet during video laryngoscope guided nasotracheal intubation. According to an article by Kumar et al, in which three types of endotracheal tubes were compared for cuff inflation technique for direct laryngoscopic guided nasal intubation and they found that cuff inflation could correctly realign $94.5 \%$ of all the malaligned endotracheal tubes. In our study, cuff inflation was used in $56.6 \%$ in group I patients and $63.33 \%$ in group II.

HK Baddoo et al studied cuff inflation to aid nasotracheal intubation using the C-MAC video laryngoscope in 5 consecutive patients needing nasotracheal intubation and found that cuff inflation of the nasotracheal tube helps direct the tube anteriorly towards the glottis, thus facilitating intubation. In our study, we found similar results in which usage of C-MAC video laryngoscope decreased the amount of time taken for intubation significantly.

AC Van Elstraete had studied the efficacy of ETT cuff inflation in the pharynx as an aid to blind nasotracheal intubation in patients with an immobilised cervical spine and the technique was compared with fibreoptic bronchoscopy, and they found that intubation was successful in 19 of 20 patients (95\%) when using fibreoptic bronchoscopy. Mean times to intubate were $20.8+/-23 \mathrm{~s}$ when the ETT cuff was inflated in the pharynx and $60.1+/-56 \mathrm{~s}$ when using fibreoptic laryngoscopy $(p<0.01)$ and they concluded that both ETT cuff inflation in the pharynx and fibreoptic bronchoscopy are valuable for nasotracheal intubation in patients with an immobilised cervical spine and that ETT cuff inflation can be used as an alternative to fibreoptic bronchoscopy in patients with CSI.

Vinuta V Patil et al studied whether C-MAC ${ }^{\circledR}$ video laryngoscope improved the nasotracheal intubating conditions compared to Macintosh direct laryngoscope in paediatric patients posted for tonsillectomy surgeries in 60 patients and found concluded that the overall performance of C-MAC ${ }^{\circledR}$ VL was better when compared to conventional direct Macintosh laryngoscope during NTI in terms of glottis visualisation, intubation time and need for additional manoeuvres.

In our study, we found that in group- $156.6 \%$ and in group-2 $63.33 \%$ patients required cuff inflation for intubation.

In group-1 $3.3 \%$ and in group-2 $6.6 \%$ patients required usage of forceps for intubation, remaining $40 \%$ and $30 \%$ in group 1 and 2 respectively required no cuff inflation for intubation.

The amount of air required for cuff inflation was $5.86 \pm$ 5.29 in Group 1 and $6.89 \pm 5.31$ in Group 2 patients.

The usage of C-MAC video laryngoscope decreased the amount of time taken for intubation significantly in Group 1 and 2 by 24.97 secs and 23.07 secs respectively.

\section{CONCLUSION}

The usage of C-MAC video laryngoscope for nasotracheal intubation of PVC and Flexometallic ETT reduced the grade of difficulty and provided easy navigability of ETT from laryngeal inlet to trachea and also decreased the amount of time taken for intubation significantly.

Limitation of this study was that anaesthesiologist could not be blinded to the type of device being used for NTI. The study was limited to only sixty patients considering intubation time as the primary outcome parameter. Larger number of enrolled cases are required to assess other parameters of the nasotracheal intubating conditions.

\section{REFERENCES}

[1] Kumar R, Gupta E, Kumar S, et al. Cuff inflationsupplemented laryngoscope-guided nasal intubation: a comparison of three endotracheal tubes. Anesth Analg 2013;116(3):619-24.

[2] Lim Y, Lim TJ, Liu EH. Ease of intubation with the GlideScope or Macintosh laryngoscope by inexperienced operators in simulated difficult airways. Can J Anaesth 2004;51(6):641-2.

[3] Low D, Healy D, Rasburn N. The use of the BERCI DCI Video Laryngoscope for teaching novices direct laryngoscopy and tracheal intubation. Anaesthesia 2008;63(2):195-201.

[4] Kaplan MB, Ward DS, Berci G. A new video laryngoscope-an aid to intubation and teaching. J Clin Anesth 2002;14(8):620-6. 
[5] Kaplan MB, Hagberg CA, Ward DS, et al. Comparison of direct and video-assisted views of the larynx during routine intubation. J Clin Anesth 2006;18(5):357-62.

[6] Enomoto Y, Asai T, Arai T, et al. Pentax-AWS, a new video laryngoscope, is more effective than the Macintosh laryngoscope for tracheal intubation in patients with restricted neck movements: a randomized comparative study. $\mathrm{Br} \mathrm{J}$ Anaesth 2008;100(4):544-8.

[7] Cooper RM, Pacey JA, Bishop MJ, et al. Early clinical experience with a new video laryngoscope (GlideScope) in 728 patients. Can J Anaesth 2005;52(2):191-8.

[8] Maassen R, Lee R, Hermans B, et al. A comparison of three video laryngoscopes: the Macintosh laryngoscope blade reduces, but does not replace, routine stylet use for intubation in morbidly obese patients. Anesth Analg 2009;109(5):1560-5.
[9] Cavus E, Thee C, Moeller T, et al. A randomised, controlled crossover comparison of the C-MAC ®video laryngoscope with direct laryngoscopy in 150 patients during routine induction of anaesthesia. BMC Anesthesiol 2011;11:6.

[10] Baddoo HK, Phillips BJ. Cuff inflation to aid nasotracheal intubation using the C-MAC video laryngoscope. Ghana Med J 2011;45(2):84-6.

[11] Van Elstraete AC, Mamie JC, Mehdaoui H. Nasotracheal intubation in patients with immobilized cervical spine: a comparison of tracheal tube cuff inflation and fiberoptic bronchoscopy. Anesthesia and Analgesia 1998;87(2):400-2.

[12] Patil VV, Subramanya BH, Kiranchand N, et al. Does CMAC ${ }^{\circledR}$ video laryngoscope improve the nasotracheal intubating conditions compared to Macintosh direct laryngoscope in paediatric patients posted for tonsillectomy surgeries? Indian J Anaesth 2016;60(10):732-6. 